\title{
High NDRG3 expression facilitates HCC metastasis by promoting nuclear translocation of $\beta$-catenin
}

\author{
JiKui Shi ${ }^{2, \#}$, HongZhen Zheng ${ }^{1, \#} \mathcal{E}$ LingYan Yuan ${ }^{1, *}$ \\ ${ }^{1}$ Department of Oncology, Changzheng Hospital, Second Military Medical University, Shanghai 200040, ${ }^{2}$ Department of Critical Care \\ Medicine, Jining NO.1 People's Hospital, Jining 272011, P.R. China
}

\begin{abstract}
NDRG1 has been reported to exert pivotal roles in tumor progression and metastasis via $\mathrm{Wnt} / \beta$-catenin signaling pathway. However, little is known about the role of NDRG3 in hepatocarcinogenesis despite its classification in the same subfamily of NDRG1. The present study was aimed to characterize the expression pattern and understand the biological roles of NDRG3 in hepatocarcinogenesis, as a means to exploit its therapeutic potential. It was observed that NDRG3 was up-regulated in HCC tissues and higher NDRG3 expression was associated with significantly shorter overall survival. Furthermore, a lower level of NDRG3 exhibited marked positive correlation with metastasis-free survival. In vitro and in vivo experiments revealed that knock-down of NDRG3 inhibits HCC metastasis and angiogenesis. We further demonstrated that activation of $\mathrm{WNT} / \beta$-catenin signaling and enhanced CSC-like properties were responsible for NDRG3mediated promoting effect on HCC. In conclusion, the principal findings demonstrated that high NDRG3 expression facilitates HCC metastasis via regulating the turnover of $\beta$-catenin, as well as provides a potential therapeutic target for future therapeutic interventions. [BMB Reports 2019; 52(7): 451-456]
\end{abstract}

\section{INTRODUCTION}

Although immense progress has been made in the treatment of hepatocellular carcinoma (HCC), disappointing prognosis still remains with a 5 -year survival rate of approximately $15-25 \%$ due to the high frequency of recurrence and metastasis after surgery $(1,2)$. Conventional chemotherapy also shows limited survival benefit due to metastasis and notorious resistance (3).

${ }^{*}$ Corresponding author. Tel: +86-21-66540109; Fax: +86-21-66540 109; E-mail: yuanlingyan@Smmu.edu.cn

${ }^{\#}$ These authors contributed equally to this work.

https://doi.org/10.5483/BMBRep.2019.52.7.201

Received 31 August 2018, Revised 19 September 2018, Accepted 31 October 2018

Keywords: $\beta$-catenin, Clinical significance, HCC, Metastasis, NDRG3
Therefore, it is extremely crucial to thoroughly investigate the molecular mechanism specifically involved in HCC metastasis and develop new targeted treatments for HCC.

N-MYC downstream-regulated gene 3 (NDRG3) (4, 5), together with NDRG1 (6), NDRG2 (4), and NDRG4 (7) constitutes the NDRG gene family and shares a conserved $\mathrm{Ndr}$ domain and an $\alpha / \beta$ hydrolase-fold region (8). NDRG family plays vital roles in the development, cancer metastasis, and the immune system (9). NDRG1, was the first to be discovered and is activated by androgens (10) and hypoxia (11). In multiply types of cancer cells, NDRG1 can act as a suppressor, by enhancing differentiation, reducing proliferation rate, and suppressing cancer metastasis $(12,13)$. Moreover, NDRG1 is necessary for p53-dependent apoptosis (14). Interestingly, in HCC, NDRG1 acts as an oncogene by directly interacting with GSK-3 $\beta$ and Nur77 to prevent $\beta$-catenin degradation (15). In general, NDRG2 is highly expressed in adult skeletal muscle and brain. Abnormal overexpression of NDRG2 is hypothesized to be associated with Alzheimer's disease pathogenesis (16). It is known that NDRG2 works as a tumor suppressor, such as in colon cancer and glioblastoma and constitutive expression of wild-type NDRG2 markedly reduces the proliferation of tumor cells $(17,18)$. The functional role of NDRG4 in cellular progression has not yet been identified. Like NDRG2, NDRG4 is highly expressed in brain and heart (9). NDRG4 functions as a tumor suppressor in colorectal cancer (19) and its role as an oncogene have been reported in glioblastoma (20).

NDRG3 is known to be involved in cell proliferation, differentiation, and other biological processes $(8,9)$. A recent study reported that NDRG3 could potentially regulate decidualization in embryo implantation via the estrogen/ progesterone/miR-290b-5p pathway (21). In male reproductive organs, such as the testis and the seminiferous epithelium, it has been hypothesized that highly expressed NDRG3 plays a role in spermatogenesis (5). In the central nervous system, NDRG3 expression is ubiquitous; whereas, NDRG1 and NDRG2 are not, which means different function during neurodevelopment (22). During the hypoxic response, cell apoptosis and cerebral ischemia are mediated by NDRG3-Raf-ERK pathway (23) and let-7f/NDRG3 pathway (24), respectively. In laryngeal squamous cell carcinoma and

ISSN: 1976-670X (electronic edition)

Copyright (C) 2019 by the The Korean Society for Biochemistry and Molecular Biology

cc) This is an open-access article distributed under the terms of the Creative Commons Attribution Non-Commercial License (http://creativecommons.org/licenses/by-nc/4.0) which permits unrestricted non-commercial use, distribution, and reproduction in any medium, provided the original work is properly cited. 
non-small cell lung cancer, NDRG3 expression is elevated, which is related to unfavorable overall survival and positive lymph node metastasis $(25,26)$. However, in breast cancer, NDRG3 is believed as a tumor suppressor candidate (27). These paradoxical reports indicate that NDRG3 expression has tissue-specific functions in various cancer types. So, what's the manner in $\mathrm{HCC}$ ?

To date, little is known about the exact role of NDRG3 in HCC. The aim of this study was to comprehensively explore the expression pattern and prognostic significance of NDRG3 in HCC. Based on the analysis of samples, we found that NDRG3 was up-regulated in tumor tissues and negatively related to prognosis. Furthermore, our data showed that NDRG3 could promote HCC metastasis by promoting nuclear translocation of $\beta$-catenin.

\section{RESULTS}

\section{Overexpression of NDRG3 in HCC tissues}

To unveil the changes in NDRG3 expression between HCC tissues and matching non-tumor tissues, 20 pairs of tumor/paracancerous tissues were used to examine NDRG3 mRNA level by qRT-PCR (Fig. 1A) and 10 pairs of those were used for western blot analysis (Fig. 1B). As shown in Fig. 1A and 1B, NDRG3 was up-regulated at both transcriptional and posttranscriptional levels in HCC. Meanwhile, similar results were verified in 156 pairs of tumor/paracancerous tissues by IHC. Stronger NDRG3 staining was detected in most of the samples (118/156, 75.64\%) (Fig. 1D), as shown in representative cases (Fig. 1C). A similar trend was observed in HCC datasets (Supplementary Fig. 1). To sum up, our data
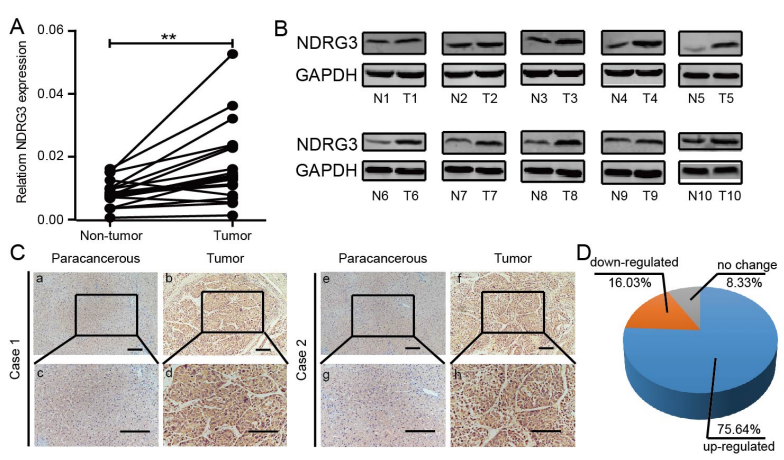

Fig. 1. NDRG3 is up-regulated in HCC tissues. (A) Relative mRNA expression of NDRG3 in $20 \mathrm{HCC}$ and matched paracancerous tissues. Values are means \pm SEM $(* * P<0.01)$. (B) Western blotting analysis of NDRG3 expression in 10 pairs of the tumor (T) and matched non-tumor (N) tissues. Glyceraldehyde-3-phosphate dehydrogenase (GAPDH) was included as a loading control. (C) Immunohistochemical staining of NDRG3 in tumor and paracancerous tissues (Original magnification: $a, b, e$, and $f 100 \times$; c, d, g and $h, 200 \times$ ). Scale bars, $50 \mu \mathrm{m}$. (D) Statistical analysis of NDRG3 expression based on immunohistochemical staining in 156 pairs of tumor/paracancerous tissues. suggested that up-regulated NDRG3 was implicated in HCC.

\section{The relationship between NDRG3 expression and clinical parameters in HCC}

Following, Chi-square test was applied to examine the clinical significance of NDRG3 in HCC. The results indicated that NDRG3 expression in HCC tissues was closely related with tumor size $(P=0.010)$, tumor encapsulation $(P=0.0081)$, liver cirrhosis $(P=0.010)$, vascular invasion $(P=0.024)$, and TNM stage $(P=0.007)$, whereas no significant differences were found between NDRG3 expression and age, gender, thromb and serum AFP (Table S1).

\section{Association of NDRG3 expression with clinical outcomes in HCC}

We subsequently investigated the clinical significance of NDRG3 in HCC. In the TCGA cohort, worse OS (overall survival) was observed frequently in HCC patients with higher NDRG3 expression (Fig. 2A). To further determine the correlation between NDRG3 and prognostic value in HCC, NDRG3 expression was detected by IHC analysis in 174 pathologist-confirmed and clinically annotated specimens, who were divided into lower and higher group depending on the staining area and intensity (Fig. 2B). Consistent with the
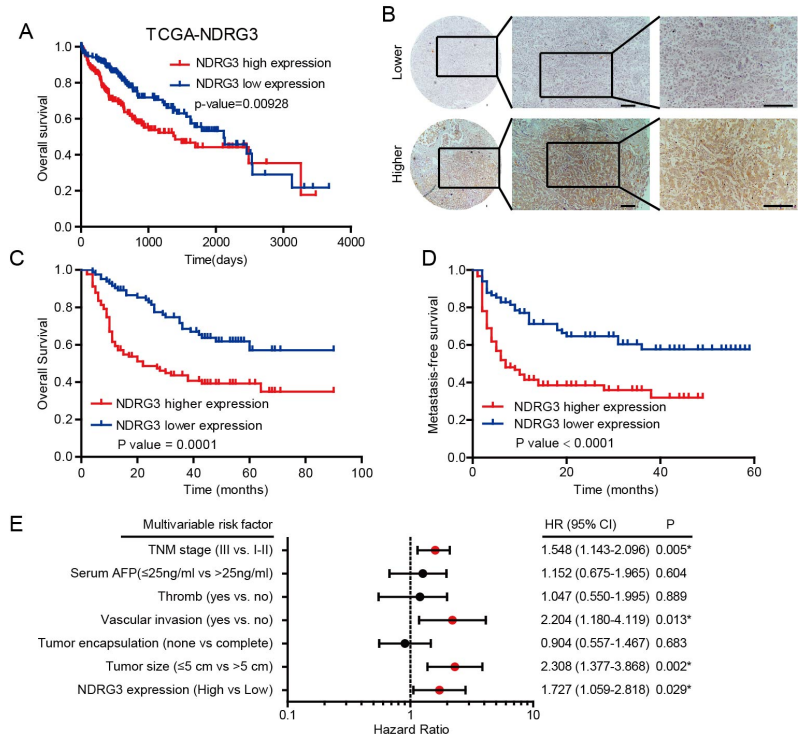

Fig. 2. The relationship between NDRG3 expression and prognosis in HCC patients. (A) Kaplan-Meier analysis of prognosis in HCC patients based on mRNA expression of NDRG3 using TCGA cohort and the median value was used as a cut-off (log-rank test, $\mathrm{P}=0.009$ ). (B) Representative images of NDRG3 in a set of tissue microarray including $174 \mathrm{HCC}$ samples. Scale bar: $50 \mathrm{~mm}$. (C, D) Kaplan-Meier analyses of the OS and MFS based on NDRG3 protein expression in $174 \mathrm{HCC}$ patients (log-rank test, $\mathrm{P}=0.0001$ and $\mathrm{P}<0.0001$, respectively). (E) Multivariate Cox regression analyses were performed in 174 HCC patients. 
results of the TCGA cohort, patients with higher NDRG3 expression were likely to have a shorter OS (Fig. 2C, P = 0.001). Interestingly, patients with lower NDRG3 levels had a significantly higher metastasis-free survival (MFS) rate compared to patients with higher NDRG3 level (Fig. 2D, P < 0.001). Collectively, these results indicate that increased NDRG3 promotes HCC metastasis in tumor progression.

Furthermore, univariate and multivariate analysis were applied using a Cox proportional hazards model to assess the correlation between NDRG3 expression and patients' prognosis. In univariate analysis, NDRG3 expression, tumor size, tumor encapsulation, vascular invasion, thromb, serum AFP, and TNM stage were hazardous prognostic factors for the overall survival (Table S2). Multivariate Cox regression analysis showed that NDRG3 expression, tumor size, vascular invasion, and TNM stage were confirmed as independent predictors of unfavorable prognosis (Fig. 2E). Herein, we concluded that elevated NDRG3 expression exert bad effect on patients' prognosis and tumor progression in HCC.

\section{NDRG3 enhances HCC metastasis and angiogenesis in vitro and in vivo}

The functional role of NDRG3 in HCC metastasis was explored. In Huh7 and HepG2 cells, we established stable NDRG3-knockdown cells by shRNA. Western blotting (Fig.

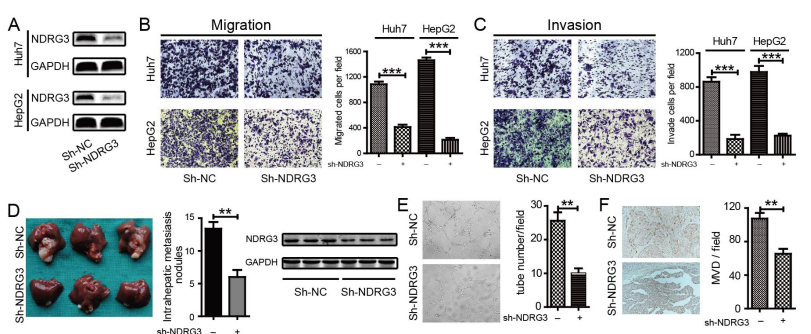

Fig. 3. Knockdown of NDRG3 impairs HCC metastasis and angiogenesis in vitro and in vivo. (A) Knockdown efficacy of NDRG3 in Huh7 and HepG2 was verified by Western Blot. (B, C) Transwell assays were performed by NDRG3-knockdown and control cells of Huh7 and HepG2 to examine the migration (B) and invasion ability (C). Quantification of migrated and invaded cells were performed from six randomly selected fields (original magnification: $200 \times$ ), values are means + SD $* * * P<0.001$. (D) Representative photographs of intrahepatic metastases in sh-NDRG3-HepG2 and control mice (5 mice in each group), along with the number of metastatic nodules and knockdown effect of NDRF3 in the liver tissues. (E) Tube formation assay showed that CM from the supernatant of sh-NDRG3-HepG2 could inhibit angiogenesis in vitro and tube number per field was measured (Original magnification: 100×; **P $<0.01$ ). (F) Representative photographs of tumor sections stained with anti-CD31 (MVD) from sh-NDRG3-HepG2 and control intrahepatic metastases. The MVD was determined by counting the number of CD31-positive vessels, values are means $\pm \mathrm{SD}, * * \mathrm{P}<0.01$.
3A) confirmed the interference effects of NDRG3 in HCC cells. As shown in Fig. 3, HCC cells with NDRG3-knockdown exhibited significantly weaker migration (Fig. 3B) and invasion (Fig. 3C) ability than the control cells in vitro. We further confirmed the inhibitory effect of NDRG3 on HCC cell metastasis in vivo. Four weeks after the liver implantation in the left hepatic lobes of nude mice with sh-NDRG3-HepG2 and sh-NC-HepG2 cells (5 in each group), more intrahepatic metastatic nodules were observed in the mice inoculated with the sh-NC-HepG2 cells, and the knockdown effect of NDRF3 in the tissues was texted by western blot (Fig. 3D).

It was apparent that angiogenesis was involved in metastasis of malignant tumor, including HCC. The results in Table S1 indicate the correlation between high expression of NDRG3 and vascular invasion $(P=0.024)$. Hence, we effect of NDRG3 on neovascularization was evident. It was observed that weaker neovascularization appeared after treatment with CM (conditional medium) from medium supernatant of sh-NDRG3-HepG2 cells compared with control counterpart (Fig. 3E). Determination of MVD (microvessel density) in an intrahepatic metastatic mouse model showed a significant decrease in the number of MVD in the sh-NDRG3-HepG2 tumor by $>40 \%$ as compared with the control counterpart (Fig. 3F). It was evident that up-regulated NDRG3 could

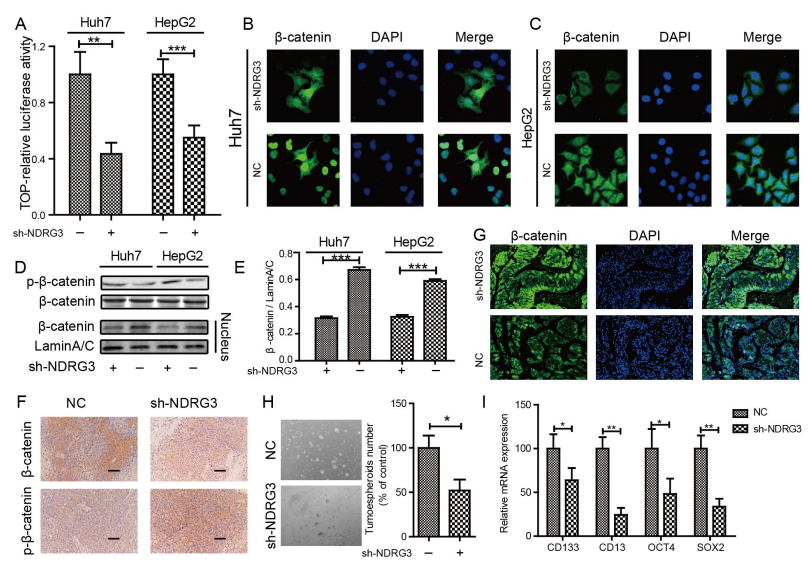

Fig. 4. NDRG 3 activates the $\mathrm{WNT} / \beta$-catenin pathway by promoting nuclear translocation of $\beta$-catenin. (A) Luciferase reporter gene assay of HCC cells treated with sh-NDRG3 or untreated cells. Values are means $\pm \mathrm{SD}$ of relative firefly/Renilla ratio, $* * \mathrm{P}<$ $0.01, * * * \mathrm{P}<0.001$. (B, C) Down-regulated NDRG3 could inhibit nuclear translocation of $\beta$-catenin in Huh7 (B) and HepG2 (C) cells as evidenced by IF staining. (D) The expression of phosphorylation of $\beta$-catenin and the amount of nuclear $\beta$-catenin was detected by Western Blotting. (E) The gray value analysis of nuclear $\beta$-catenin in NDRG3-knockout and control cells. (F) IHC staining of $\beta$-catenin and $\mathrm{p}$ - $\beta$-catenin in intrahepatic metastases tissues. Scale bars: $50 \mu \mathrm{m}$. (G) IF staining of $\beta$-catenin distribution in intrahepatic metastases tissues. $(\mathrm{H})$ Knock-down of NDRG3 inhibited spheroids formation of Huh7 cells. (I) Knock-down of NDRG3 decreased the expression of stem cell markers, CD133, CD13, OCT4, and SOX2. 
enhance metastasis and angiogenesis in the progression of HCC.

\section{NDRG3 activates the $W N T / \beta$-catenin pathway by promoting nuclear translocation of $\beta$-catenin and promotes CSC-like properties of HCC cell in vitro}

WNT/ $\beta$-catenin has been reported to play an important role in mediating the biological behavior of NDRG1 (15). As an important paralog of NDRG1, WNT/ $\beta$-catenin may exhibit significant role in NDRG3. To uncover the underlying mechanism of NDRG3-mediated inhibition of HCC metastasis, a dual-luciferase reporter gene assay was used to verify whether NDRG3 can activate the canonical WNT/ $\beta$-catenin pathway. The reporter activity was significantly inhibited in the NDRG3 knockdown group (Fig. 4A). Subsequently, IF staining was carried out and the results showed that apparently fewer $\beta$-catenin was located in the nuclear region in NDRG3-knockdown Huh7 (Fig. 4B) and HepG2 (Fig. 4C) cells as compared with the control cells. A similar phenomenon was observed by Western Blotting in nucleoprotein detection (Fig. 4D and 4E). Moreover, enhanced phosphorylation of $\beta$-catenin was observed when NDRG3 was down-regulated in HCC cells (Fig. 4D) and mouse tissues (Fig. 4F). Therefore, we speculated that NDRG3 could prevent degradation to promote nuclear translocation of $\beta$-catenin. Next, we examined the distribution of $\beta$-catenin in liver tissues in an intrahepatic metastatic xenograft mouse model of HCC. The results revealed weaker content and nuclear localization of $\beta$-catenin in the NDRG3-knockdown group than in the control group by IHC (Fig. 4F) and IF (Fig. 4G).

It is well known that $\beta$-catenin signaling is involved in HCC stemness. Spheroid formation assays were performed to examine the CSC-like properties of HCC cell. Spheroid formation was significantly suppressed in Huh7 cells down-regulating NDRG3 (Fig. 4H). Knock-down of NDRG3 also resulted in a significant decrease in stem cell markers, such as CD133, CD13, OCT4, and SOX2 (Fig. 4I). These data suggest that NDRG3 could promote nuclear translocation of $\beta$-catenin and CSC-like properties in HCC.

\section{DISCUSSION}

In this study, we comprehensively described the expression pattern of NDRG3 in HCC and firstly demonstrated that high expression of NDRG3 was associated with unfavorable prognosis in HCC patients. Increased NDRG3 expression was closely related to tumor size, tumor encapsulation, liver cirrhosis, vascular invasion, and TNM stage. It is hypothesized that NDRG3 may promote HCC malignant behavior, which is consistent with the previous reports in the laryngeal squamous cell carcinoma and non-small cell lung cancer $(25,26)$. The results above have showed statistical significance between NDRG3 expression and the OS. Thus, we speculated that NDRG3 can be used as a predictor for the OS as well as an independent prognostic factor in HCC patients. Besides, it is intriguing to note that increased NDRG3 expression was negatively related to MFS. Accordingly, the manner in which NDRG3 works to regulate tumor cell metastasis remains unanswered.

Phylogenic analysis demonstrated that human NDRG1 and NDRG3 belong to one subfamily. Growing evidence suggests that NDRG1 is implicated in tumor metastasis. In human prostate cancer, NDRG1, together with KAI1, concerted metastasis-suppressive activities through ATF3-NFkappaB complex (28). Liu et al. found that NDRG1 could weaken the metastasis ability of tumor by inhibiting c-Src cascade signaling pathway (29). In human colorectal cancer, NDRG1 inhibits EMT, migration, and invasion through ubiquitylation and degradation of caveolin-1 (30). In HCC, up-regulated NDRG1 enhances portal vein invasion and intrahepatic metastasis (31), as well as promotes cell growth by directly interacting with GSK-3 $\beta$ and Nur77 to prevent $\beta$-catenin degradation (15). Moreover, $\beta$-catenin could be mediated by miR-31, and NDRG3 was one of the targets of miR-31 (32). Hence, it is hypothesized that $\mathrm{Wnt} / \beta$-catenin signal may be involved in NDRG3-mediated HCC metastasis. In the present study, in vitro, and in vivo assays verified that NDRG3 promotes metastasis by inhibiting degradation of $\beta$-catenin to facilitate nuclear translocation. Moreover, it is well known that $\beta$-catenin signaling is involved in HCC stemness. It was apparent that knock-down of NDRG3 inhibited the CSC-like properties of HCC cells in vitro revealing that inhibition of NDRG3 expression influenced stemness. However, the manner in which NDRG3 regulates $\beta$-catenin and CSC-like properties remain unknown and the relationship between NDRG3, self-renewal of cells and $\beta$-catenin signaling demand further investigations.

In summary, our data firstly revealed that frequent upregulation of NDRG3 expression unfavorably impacts the survival of HCC patients due to its potential of promoting metastasis by inhibiting degradation of $\beta$-catenin to facilitate nuclear translocation and enhanced CSC-like properties. This study also provides new insight into the pro-tumor role of NDRG3 in HCC. It is proposed that NDRG3 can be employed as a potential target in developing anticancer strategies.

\section{MATERIAIS AND METHODS}

\section{Clinical tissue samples}

All tissue samples used in this study were obtained from patients who underwent surgical treatment between January 2004 and December 2010 in the Department of Oncology of Changzheng Hospital, Second Military Medical University. Informed consents had been signed by all the patients. The study was approved by the Research Ethics Committee of Changzheng Hospital and carried out in accordance with ethical standards as formulated in the Helsinki Declaration of 1975 (revised 1983). None of the patients had received any 
radiotherapy or chemotherapy prior to surgery. The follow up was ended on December 2013, and median survival time was 31 months (range 2-90 months).

\section{Western blotting}

Whole cell lysates were resolved by SDS-PAGE and transferred to nitrocellulose (NC) membranes (Millipore, HATF00010) using standard techniques. Primary antibodies used for Western blotting were as follows: NDRG3 (Abcam, 1:1000), GAPDH (1:10,000; Abcam).

\section{Cell culture}

Human HCC cell lines, HepG2 and Huh7, purchased from Cell Bank of the Chinese Academy of Sciences (Shanghai, China), were cultured in DMEM medium, containing $10 \%$ fetal bovine serum (FBS). Cells were cultured at $37^{\circ} \mathrm{C}$ with $5 \%$ $\mathrm{CO}_{2}$.

\section{Statistical analyses}

The chi-square test or Student's t-test was used for comparison between groups by GraphPad Prism 5 software. Statistical significance was accepted at $\mathrm{P}<0.05$.

\section{ACKNOWLEDGEMENTS}

We are thankful to all the authors for their assistance with the experiments and providing constructive comments on the manuscript.

\section{CONFLICTS OF INTEREST}

The authors have no conflicting interests.

\section{REFERENCES}

1. Altekruse SF, Henley SJ, Cucinelli JE and McGlynn KA (2014) Changing hepatocellular carcinoma incidence and liver cancer mortality rates in the United States. Am J Gastroenterol 109, 542-553

2. Kim SR, Kudo M, Hino O et al (2008) Epidemiology of hepatocellular carcinoma in Japan and Korea. A review. Oncology 75 Suppl 1, 13-16

3. Zhu AX (2010) Systemic treatment of hepatocellular carcinoma: dawn of a new era? Ann Surg Oncol 17, 1247-1256

4. Okuda $\mathrm{T}$ and Kondoh $\mathrm{H}$ (1999) Identification of new genes ndr2 and ndr3 which are related to Ndr1/RTP/Drg1 but show distinct tissue specificity and response to N-myc. Biochem Biophys Res Commun 266, 208-215

5. Zhao W, Tang R and Huang Y (2001) Cloning and expression pattern of the human NDRG3 gene. Biochim Biophys Acta 1519, 134-138

6. Shimono A, Okuda T and Kondoh H (1999) N-mycdependent repression of ndr1, a gene identified by direct subtraction of whole mouse embryo cDNAs between wild type and N-myc mutant. Mech Dev 83, 39-52

7. Zhou RH, Kokame K, Tsukamoto Y, Yutani C, Kato $\mathrm{H}$ and
Miyata T (2001) Characterization of the human NDRG gene family: a newly identified member, NDRG4, is specifically expressed in brain and heart. Genomics 73, 86-97

8. Qu X, Zhai Y, Wei $\mathrm{H}$ et al (2002) Characterization and expression of three novel differentiation-related genes belong to the human NDRG gene family. Mol Cell Biochem 229, 35-44

9. Melotte V, Qu X, Ongenaert M et al (2010) The N-myc downstream regulated gene (NDRG) family: diverse functions, multiple applications. FASEB J 24, 4153-4166

10. Ulrix W, Swinnen JV, Heyns W and Verhoeven G (1999) The differentiation-related gene 1, Drg1, is markedly upregulated by androgens in LNCaP prostatic adenocarcinoma cells. FEBS Lett 455, 23-26

11. Lee GY, Chun YS, Shin HW and Park JW (2016) Potential role of the N-MYC downstream-regulated gene family in reprogramming cancer metabolism under hypoxia. Oncotarget 7, 57442-57451

12. Bandyopadhyay S, Wang Y, Zhan R et al (2003) The Drg-1 gene suppresses tumor metastasis in prostate cancer. Cancer Res 63, 1731-1736

13. Guan RJ, Ford HL, Fu Y, Li Y, Shaw LM and Pardee AB (2000) Drg-1 as a differentiation-related, putative metastatic suppressor gene in human colon cancer. Cancer Res 60, 749-755

14. Stein S, Thomas EK, Herzog B et al (2004) NDRG1 is necessary for p53-dependent apoptosis. J Biol Chem 279, 48930-48940

15. Lu WJ, Chua MS, Wei W and So SK (2015) NDRG1 promotes growth of hepatocellular carcinoma cells by directly interacting with GSK-3OI and Nur77 to prevent OI-catenin degradation. Oncotarget 6, 29847-29859

16. Mitchelmore C, Büchmann-Møller S, Rask L, West MJ, Troncoso JC and Jensen NA (2004) NDRG2: a novel Alzheimer's disease associated protein. Neurobiol Dis 16, 48-58

17. Deng Y, Yao L, Chau L et al (2003) N-Myc downstream-regulated gene 2 (NDRG2) inhibits glioblastoma cell proliferation. Int J Cancer 106, 342-347

18. Kim YJ, Yoon SY and Kim JT (2009) NDRG2 suppresses cell proliferation through down-regulation of AP-1 activity in human colon carcinoma cells. Int J Cancer 124, 7-15

19. Melotte V, Lentjes MH, van den Bosch SM et al (2009) $\mathrm{N}-\mathrm{Myc}$ downstream-regulated gene 4 (NDRG4): a candidate tumor suppressor gene and potential biomarker for colorectal cancer. J Natl Cancer Inst 101, 916-927

20. Schilling $\mathrm{SH}$, Hjelmeland $A B$, Radiloff $D R$ et al (2009) NDRG4 is required for cell cycle progression and survival in glioblastoma cells. J Biol Chem 284, 25160-25169

21. Yang Q, Zhang X, Shi Y et al (2017) Increased Expression of NDRG3 in Mouse Uterus During Embryo Implantation and in Mouse Endometrial Stromal Cells During In Vitro Decidualization. Reprod Sci 25, 1197-1207

22. Okuda T, Kokame K and Miyata T (2008) Differential expression patterns of NDRG family proteins in the central nervous system. J Histochem Cytochem 56, 175-182

23. Cui C, Lin H, Shi $Y$ and Pan R (2017) Hypoxic postconditioning attenuates apoptosis via inactivation of 
adenosine A2a receptor through NDRG3-Raf-ERK pathway. Biochem Biophys Res Commun 491, 277-284

24. Yao Y, Wang W, Jing $L$ et al (2017) Let-7f Regulates the Hypoxic Response in Cerebral Ischemia by Targeting NDRG3. Neurochem Res 42, 446-454

25. Luo X, Hou N, Chen X et al (2017) High expression of NDRG3 associates with unfavorable overall survival in non-small cell lung cancer. Cancer Biomark 21, 461-469

26. Ma J, Liu S, Zhang W et al (2016) High expression of NDRG3 associates with positive lymph node metastasis and unfavourable overall survival in laryngeal squamous cell carcinoma. Pathology 48, 691-696

27. Estiar MA, Zare AA, Esmaeili R et al (2017) Clinical significance of NDRG3 in patients with breast cancer. Future Oncol 13, 961-969

28. Liu W, liizumi-Gairani M, Okuda $\mathrm{H}$ et al (2011) KAl1 gene is engaged in NDRG1 gene-mediated metastasis suppression through the ATF3-NFkappaB complex in human prostate cancer. J Biol Chem 286, 18949-18959

29. Liu W, Yue F, Zheng M et al (2015) The proto-oncogene c-Src and its downstream signaling pathways are inhibited by the metastasis suppressor, NDRG1. Oncotarget 6, 8851-8874

30. Mi L, Zhu F, Yang X et al (2017) The metastatic suppressor NDRG1 inhibits EMT, migration and invasion through interaction and promotion of caveolin-1 ubiquitylation in human colorectal cancer cells. Oncogene 36, 4323-4335

31. Akiba J, Ogasawara S, Kawahara A et al (1994) N-myc downstream regulated gene 1 (NDRG1)/Cap43 enhances portal vein invasion and intrahepatic metastasis in human hepatocellular carcinoma. Oncol Rep 20, 1329-1335

32. Du Z, Niu S, Xu X and Xu Q (2017) MicroRNA31-NDRG3 regulation axes are essential for hepatocellular carcinoma survival and drug resistance. Cancer Biomark 19, 221-230 